\title{
Sustainable scenarios for and in university education: design of sustainable products
}

\author{
Julián Fernando León Durango Gómez \\ Instituto Tecnológico Metropolitano | Colombia | juliandurango@itm.edu.co \\ Maria Isabel Giraldo Vásquez \\ Instituto Tecnológico Metropolitano | Colombia | mariavasquez@itm.edu.co
}

\begin{abstract}
This paper proposes instruments from the sustainable development framework for the construction of a sustainable product design undergraduated program guide. The methodology used was mixed: surveys, both quantitative and qualitative, focus groups and semi-structured virtual interviews were carried out. Information on the state of the art was collected and later served as input for the construction of the guide. The results showed a concern about the lack of academic spaces to train with skills and conscience about the topic. Likewise, these allowed the construction of this guide, where knowledge that promotes more sustainable consumption and production could be imparted.
\end{abstract}

Keywords: Sustainable design; Curriculum; Undergraduated degree; Academic instruments.

\section{INTRODUCCIÓN}

La idea de integrar la conciencia del desarrollo sostenible en el pensamiento, teoría y práctica del diseño (en general y en particular del diseño de productos) es una preocupación y una necesidad cada vez más apremiante: según la ONU (Naciones Unidas, 2017), las cifras mundiales apuntan a que el consumo total de recursos naturales utilizados en procesos económicos aumentó a $1.3 \mathrm{~kg}$ por unidad del PIB entre 2000 y 2010 . En Colombia, el consumo interno de materiales total también aumentó durante el mismo período, de 48.700 millones de toneladas a 71.000 millones (DANE, 2017)

Los diseñadores desempeñan un papel clave en la evolución de las prácticas de producción, comercialización y adquisición de productos, porque deben tomar decisiones cruciales y dar solución a problemáticas respecto a los materiales, la fabricación, los insumos y la comercialización de los bienes de consumo, todas estas decisiones implican impactos de carácter global. A nadie se le escapan las múltiples interrelaciones entre el consumo humano, desarrollo económico y medio ambiente (Delgado-Huertos, 1998). La idea de que se puede vivir bien consumiendo menos recursos y generando un sentido de comunidad se opone completamente al modelo que hasta ahora ha prevalecido en la sociedad industrial. Por ello, no es posible dar un paso significativo hacia la sostenibilidad a partir de las ideas y métodos actuales. (Manzini, Design, ethics and sustainability Guidelines for a transition phase, 2006). Desde la perspectiva de la enseñanza del diseño industrial, nuevos paradigmas, prácticas, formas de producción y consumo llevan a repensar los espacios para el desarrollo académico, en donde se permita la formación de ciudadanos con habilidades y conciencia para desenvolverse en la contemporaneidad, y pensar una formación de ciudadanía más sostenible. Aunque el diseño de un producto tiende a tener en cuenta el ciclo de vida, todavía no recibe la atención adecuada con respecto a la sostenibilidad. La atención se sigue centrando en gran medida en la mejora de producción, la eficiencia y la rentabilidad económica. Así pues, el Diseño, como disciplina actual, debe reformularse, reconstruirse y proyectar una nueva alternativa a la producción serializada de productos, ya que según el Environmental Change Institute (2005) el 80\% de la contaminación ambiental y el $90 \%$ de los costes de fabricación son el resultado de decisiones tomadas en la etapa de diseño del producto, siendo entonces, los diseñadores posibles agentes de cambio en la economía mundial. La preocupación por generar espacios para e desarrollo académico, productivo, de pensamiento, formando personas con habilidades y conciencia para desenvolverse en el mundo actual y futuro, permite pensar en la creación y el desarrollo de un programa profesional universitario en Diseño Sostenible

\section{METODOLOGÍA}

La propuesta se desarrolló bajo una metodología mixta, en donde se realizaron en una primera etapa encuestas para toma de datos específicos tanto de carácter cuantitativo como cualitativo. Particularmente los datos cualitativos representan gran parte del ejercicio analítico pues se discutieron asuntos que tenían que ver más con la percepción de los entrevistados. (diseñadores profesionales, empresarios, académicos y estudiantes de diseño).

En una segunda etapa se realizaron grupos focales y se realizaron entrevistas semiestructuradas en donde se recolectó información sobre estado del arte frente al tema y que posteriormente sirvieron de insumo para medir la factibilidad de un programa académico con características de sostenibilidad y la construcción de una guía que sirva como apoyo a la hora de crear los contenidos curriculares, la pertinencia y la justificación de un programa profesional universitario en Desarrollo Sostenible. 


\section{DISCUSIÓN}

\section{SOBRE LOS ESTUDIOS DE TENDENCIA DE LA FORMACIÓN}

Se puede observar que existen diversas universidades que ofrecen dentro del campo del diseño, formación específica en Diseño Industrial. Este pregrado es el de mayor semejanza al programa que se pretende formular con esta investigación, por lo tanto, es al que se le han hecho los análisis correspondientes. El estado de la formación en el mundo da muestra de un incremento cada vez mayor en desarrollar prácticas académicas que impacten la industria, la producción y el consumo. Hasta el momento de finalización de esta investigación, los programas académicos de formación en Diseño Sostenible encontrados se han desarrollado a nivel posgradual, con algunos acercamientos a nivel de formación profesional. Esto podría indicar una necesidad en la formación académica de base, desarrollando perfiles de profesionales capacitados en estas áreas.

Mediante el análisis de los pensum académicos de los programas afines, se encuentra que hay una necesidad grande de formación de base en este campo específico, dado que no existe un programa con estas características a nivel profesional, quedando en evidencia que todos los programas existentes son de carácter posgradual. Por ello se plantea la posibilidad de un sistema de formación en pregrado con características que busquen desarrollar e impartir conocimientos bajo el esquema del desarrollo sostenible que promuevan un consumo y una producción más sostenible, teniendo en cuenta las tres dimensiones típicas necesarias para esto: ambiental, económica y social y contemplando una cuarta que tiene que ver con la toma de decisiones y la gobernanza.

Adicionalmente se revisaron cinco mallas académicas de programas locales con relevancia y concordancia con el objeto de estudio del programa a desarrollar en esta investigación; estos fueron: Diseño Industrial en la Universidad Pontificia Bolivariana (UPB, 2017), Diseño Industrial en la Universidad de San Buenaventura (USB Medellín, 2017), Ingeniería en Diseño Industrial en el Instituto Tecnológico Metropolitano (ITM, 2017), Ingeniería de Diseño de Producto de la Universidad EAFIT (EAFIT, 2017) y Diseño Y Gestión Del Producto de la Universidad de Medellín (UdeM, 2018). En ninguno de ellos se evidencia una inclinación hacia la sostenibilidad, teniendo pocas o nulas asignaturas que aborden estos temas. Es importante mencionar que en el ámbito internacional hay un fuerte movimiento por la reinterpretación de los elementos que intervienen en el desarrollo de políticas educativas en el diseño. A pesar de las iniciativas mundiales, los desarrollos a nivel académico de programas de pregrado en Diseño Sostenible aún son incipientes, dejando la mayoría de estos para niveles posgraduales: Especializaciones o Maestrías. El estado de la formación en el mundo da muestra de un incremento cada vez mayor en desarrollar prácticas académicas que impacten la industria, la producción y el consumo. Como se ha mencionado anteriormente, se encuentra que hay una necesidad en la formación de base en este campo específico, dado que no existe un programa con estas características a nivel profesional, quedando en evidencia que todos los programas existentes son de carácter posgradual.

\section{MEDICIÓN DE LA NECESIDAD DEL MEDIO ACADÉMICO Y PRODUCTIVO DE PROGRAMAS PROFESIONALES EN DISEÑO SOSTENIBLE}

Se planteó la necesidad de desarrollar una serie de entrevistas a profesionales que estuvieran involucrados en el ámbito del diseño industrial o diseño de productos, tanto en el sector académico, como en el sector productivo. Igualmente se realizaron encuestas a estudiantes de diseño industrial, ingeniería en diseño industrial y similares. Ambos ejercicios permitieron conocer la percepción del medio académico y productivo acerca del Diseño Sostenible como disciplina y la necesidad o no, de tener programas de formación académica especializados en estas áreas. Para medir la percepción en estudiantes se utilizó el método de la encuesta (Hernández-Sampieri, 2014), que permite obtener resultados masivos de forma fácil. Para ello se ha conformado una selección de preguntas que se utilizan en la medición de variables. De esta manera se registraron de forma sencilla y práctica datos de interés que se pretendían recolectar. Se ha utilizado un tipo de encuesta mixta, en donde los participantes encontraron 16 preguntas en total de las cuales 12 eran de opción cerrada y las restantes cuatro abiertas, donde podian expresar abiertamente sus opiniones frente a las preguntas que se hacían en la encuesta. Las preguntas realizadas en la encuesta fueron las siguientes:

\section{-Género}

-Edad

-Está graduado del pregrado?

-Sabe usted que es diseño sostenible?

-En su programa académico, el diseño sostenible está inmerso en el currículo?

-Le interesaría conocer o estudiar del tema? - ¿Le gustaría que el pénsum de una carrera a nivel profesional universitario tuviera este énfasis o enfoque? -La enseñanza de la sostenibilidad al interior de los programas de diseño está acorde a las demandas del mercado académico y laboral?

-Cree usted que la enseñanza del Diseño Sostenible prepararía a profesionales que respondan a las necesidades sociales de su ciudad/región?

-Cree usted que la enseñanza del diseño está actualizada en función de las tendencias y necesidades ambientales y para la sostenibilidad?

En cuanto a las entrevistas realizadas a los profesionales, se utilizó un formato estructurado donde, siguiendo una guía de preguntas específicas (Hernández-Sampieri, 2014) y sujetas exclusivamente a éstas, los participantes expresaron abiertamente sus opiniones. Teniendo en cuenta las características de la investigación en curso, se usaron las entrevistas como herramientas para recolectar datos cualitativos, debido a la complejidad del tema y las posibles variantes que se podrían expresar en ellas. Sin embargo, gracias a que las entrevistas se enviaron por medio electrónico, se dio pie a que los participantes se expresaran libremente sin la premura del tiempo o la presión de tener al entrevistador frente a frente.

Tanto las respuestas a la encuesta como a las entrevistas son muy similares, por lo menos en la concepción y la importancia que se le ve al Diseño Sostenible, a la necesidad de este en el ámbito productivo y son conscientes que para que esto llegue a pasar, la 
enseñanza de esto no puede ser solo en niveles posgraduales, ni debe dejarse a que algunos pocos estudiantes quieran aprender de procesos para desarrollar productos más sostenibles. Los entrevistados aseguran que hay una necesidad grande de profesionales en este campo y que se debe tener un énfasis en la sostenibilidad en los programas de diseño, no dejarlo a unas cuantas materias o líneas dentro de los programas. Estas afirmaciones, junto con lo recogido en las encuestas da luces acerca del camino que se debe tomar para plantear herramientas para la creación de programas académicos que cumplan con estas necesidades y que satisfagan el mercado laboral y empresarial, sin sacrificar el asunto medioambiental, sino por el contrario, respetarlo y a través de sus buenas prácticas como profesionales, cuidarlo y mejorar la calidad de vida de los usuarios de sus productos.

\section{HALLAZGOS}

La evaluación del estado actual de la formación en el área específica, en donde se realizó un rastreo nacional e internacional de programas con afinidad al que se busca plantear arroja en el registro nacional un total de 31 programas de formación profesional universitaria con denominación Diseño Industrial y 108 programas académicos que tienen que ver con el concepto de Sostenibilidad, sin embargo, 20 de ellos están inactivos. En general se pudo hallar que el área de la sostenibilidad está claramente vinculada a la formación posgradual, encontrando cuatro ofertas a nivel de doctorado, 18 especializaciones tecnológicas, especializaciones universitarias 20 y en Maestría 24, para un total de 66 programas de posgrado, correspondiente al $75 \%$. Mientras que el restante $25 \%$ se distribuye en Formación Técnica Profesional con siete programas, en Tecnológica 13 y en Universitaria Profesional solo dos, ninguno de los cuales tiene que ver con el área del diseño.

Se encuentra también que solo dos programas contienen en su denominación el término Diseño Sostenible, ambos en nivel de maestría y solo uno de ellos está realmente enfocado desde su currículo al diseño de productos sostenibles, el otro hace referencia a diseño arquitectónico, lo cual confirma la tendencia encontrada, tanto dentro como fuera del país, donde el tema de Diseño Sostenible se entiende en su mayoría como diseño arquitectónico, urbano o del paisaje.

Esto también confirma una de las premisas con las que da inicio esta investigación, en la que se planteaba la falta de oferta de formación de base en el campo del Diseño Sostenible, principalmente en temas de diseño de productos. Así mismo, teniendo en cuenta la oferta actual de programas en diseño industrial, ingeniería en diseño y similares, se hace un análisis de sus planes de estudio encontrando que en ninguno de ellos se evidencia una inclinación marcada hacia la sostenibilidad, teniendo pocas asignaturas que aborden estos temas.

Estos hallazgos en las etapas primarias de la investigación, permitieron continuar con la exploración de las posibilidades y formular preguntas frente a la falta de oferta para medir a través de entrevistas y encuestas la necesidad de formación de profesionales en el área planteada.

Para ello se recurrió a encuestas a estudiantes de carreras afines, en donde la gran conclusión hace referencia a lo hallado en el punto anterior, reafirmando que el componente de sostenibilidad no hace parte del núcleo central de los programas académicos en los que estudian. Así mismo se logra evidenciar que si bien no hay una formación específica, ni un amplio conocimiento al respecto, hay una fuerte inclinación por parte de los estudiantes entrevistados por ampliar sus conocimientos en esta área, reconociendo que es importante, debido a las necesidades actuales del sector productivo, la sociedad y el ambiente.

Por otra parte, las entrevistas a los profesionales, diseñadores, empresarios, docentes e investigadores del área específica, ratifican la necesidad de generar estrategias de formación académica que impliquen un conocimiento profundo en el tema del Diseño Sostenible, ya que es hacia donde se debería encaminar la disciplina. Se manifiesta que es importante crear conciencia y pensar en un programa de Diseño Sostenible que abarque todos los ámbitos y etapas del producto o servicio y la necesidad de que todas las expresiones del diseño se enmarquen en el esquema de la sostenibilidad. La educación de diseño en general debe ser redirigida para fortalecer el desarrollo de un diseñador ético que asuma la responsabilidad de las consecuencias de la interacción humana con los productos, bienes y servicios concebidos y producidos para cada día diseñar productos más limpios, éticos y que no solo no sean perjudiciales para el medio ambiente y para la calidad de vida de las personas, sino que mejoren ambos aspectos.

\section{CONSTRUCCIÓN DE LOS PARÁMETROS NECESARIOS PARA LA FORMULACIÓN DE UN PROGRAMA PROFESIONAL EN DISEÑO SOSTENIBLE}

Luego de los hallazgos encontrados, se estableció el desarrollo de los elementos característicos de un programa de formación académica. Para ello se elaboró un constructo teórico apoyado en literatura científica, en la que se encuentran textos clásicos del diseño, autores, investigadores y diseñadores reconocidos mundialmente y artículos de investigación actuales. Todo ello sirve de material de apoyo para dar forma y sustento a lo planteado. En este sentido y teniendo en cuenta las condiciones de calidad del MEN (MEN, 2019) y el alcance de este proyecto, se han desarrollado los siguientes puntos:

\section{- Denominación del programa \\ Justificación \\ Contenidos Curriculares: componentes teóricos y componentes pedagógicos (de manera parcial).}




\section{DENOMINACIÓN DEL PROGRAMA}

Efectivamente el objeto de estudio del programa académico que se considere formular basado en esta guía, se enmarca dentro de la definición anterior (diseño industrial), ya que se centrará la formación en la enseñanza-aprendizaje de la forma, función y procesos (entre otros), todo desde un punto de vista sostenible, el asunto de la configuración morfológica, el uso de objetos, la producción y las interacciones con los usuarios y sus contextos son de fundamental y esencial aprehensión dentro de este campo disciplinar. De acuerdo a lo anterior y teniendo en cuenta la normativa, las reglamentaciones y los lineamientos que el MEN aporta para la construcción de programas académicos, se podría contemplar que en este sentido la denominación de un programa basado en las condiciones que en este texto se han enunciado, podría variar entre Diseño de Producto Sostenible, Diseño de Objeto Sostenible o simplemente Diseño Sostenible. Es evidente la pertinencia y coherencia con el termino Diseño y por ello no se ahondará en la definición del concepto; será suficiente con definir que el diseño se concibe desde la mirada del Ministerio de Educación como:

"un proceso prospectivo, proyectual y creativo para la creación de soluciones centradas en los seres humanos. Articula habilidades intelectuales, conceptos, conocimientos, destrezas y métodos para integrar las características morfológicas, culturales, productivas, comunicativas y estéticas del proyecto. Las soluciones de diseño satisfacen de manera sustentable necesidades y deseos, con el concurso de los actores involucrados en contextos determinados." (MEN, 2010).

Sin embargo, el asunto de lo Sostenible, que es fundamental para esta guía, se concibe como un objetivo alcanzable a través de múltiples factores y que tendrá que ser definido para justificar la denominación seleccionada.

La definición de sostenibilidad más citada se deriva del Informe de la Comisión Brundtland de 1987, desarrollado en el marco del documento denominado Nuestro Futuro Común que describe al Desarrollo Sostenible como un "desarrollo que satisface las necesidades del presente sin comprometer la capacidad de las generaciones futuras para satisfacer sus propias necesidades " (UN, 1987).

\section{JUSTIFICACIÓN}

Una de los argumentos más importantes que se pueden dar a la hora de justificar la importancia de crear un programa en Diseño Sostenible, es la necesidad de integrar la formación en desarrollo sostenible en el pensamiento, teoría y práctica del diseño (en general y en particular del diseño de productos, de objetos), esta es una preocupación y una necesidad cada vez más apremiante; según la ONU (Naciones Unidas, 2017; Naciones Unidas, 2017). Así pues, el método tradicional de creación, producción y comercialización de los productos no puede seguir siendo el estándar. Este enfoque está siendo desafiado por los nuevos tipos de consumidores. Este es uno de los campos donde el diseño entra a formar parte crucial en la ecuación de la producción / comercialización / consumo. Los diseñadores desempeñan un papel clave en la evolución de las prácticas de producción, comercialización y adquisición de productos, porque deben tomar decisiones cruciales y dar solución a problemáticas respecto a los materiales, la fabricación, los insumos y la comercialización de los bienes de consumo, todas estas decisiones implican impactos de carácter global. Tal como lo plantea Delgado-Huertos (1998), a nadie se le escapan las múltiples interrelaciones entre el consumo humano, el desarrollo económico y el medio ambiente (DelgadoHuertos, 1998). El diseño ha tenido que hacer cambios en sus maneras de producir; en el caso de lo que plantea la economía circular, el diseño de productos debe buscar extender la vida útil de los mismos (Bakker, den Hollander, Van Hinte, \& Zljlstra, 2014). Pero en última instancia, el papel del diseño debe ir un paso más allá. Este siempre ha sido generar valor y bienestar, de allí que, en el momento histórico actual, el diseño debe pasar a ser aún más estratégico desarrollando lo que Manzini define como 'Diseño Estratégico para la Sostenibilidad', esto es:

"diseño de una estrategia de innovación, cambiando el enfoque comercial del diseño (y la venta) solo de productos físicos, para diseñar (y vender) un sistema de productos y servicios que sean conjuntamente capaces de satisfacer las demandas específicas de los clientes, mientras reorientan las tendencias actuales no sostenibles en las prácticas de producción y consumo". (Manzini \& Vezzoli, 2003).

La preocupación por generar espacios para el desarrollo académico, productivo, de pensamiento, formando personas con habilidades y conciencia para desenvolverse en el mundo actual y futuro, permite pensar en la necesidad de creación y desarrollo de programas profesionales universitarios en Diseño Sostenible.

El objetivo de cualquier diseñador, al margen de su opinión sobre el Diseño Sostenible, es crear un objeto duradero. "Que un producto se perpetúe a lo largo del tiempo es la mejor prueba de su diseño ingenioso y elegante" (Mallory \& Ohlman, 2005). Las condiciones son propicias y están a favor para lograr desarrollar esta clase de programas académicos. Lograr este tipo de transformaciones permitiría acercarse a la meta global del desarrollo sostenible de la Comission on Environment and Development (CED) que, en 1987, enfatizaba en la premisa de conseguir un progreso que satisficiera las necesidades del presente sin comprometer la capacidad de las futuras generaciones de satisfacer sus propias necesidades, para minimizar la degradación que la industria causa en el medio ambiente. Para ello se deberán realizar mayores esfuerzos empresariales y políticos. Por tanto, deberán transformarse las tradicionales prácticas industriales, orientándose desde un sistema autorreferencial, abierto, lineal y generador de residuos, hacia otro mucho mayor, cerrado y cíclico que ahorre recursos e incorpore a la naturaleza como nuevo interlocutor (Chambouleyron, Arena, \& Pattini, Diseño de productos y desarrollo sustentable, 2000).

Dadas las circunstancias que se han mencionado, tanto en lo global en los campos social, medioambiental y económico, como en lo local, en el ámbito de la formación académica, es importante destacar el papel del diseñador para consolidar el cambio de paradigmas dentro del diseño enfocado a la sostenibilidad, siendo necesario el conocimiento de los diseñadores para motivar y orientar a la comunidad para identificar sus necesidades y generar soluciones trabajando bajo la misma visión común de un futuro sostenible. 
CONTENIDOS CURRICULARES- COMPONENTES TEÓRICOS Y COMPONENTES PEDAGÓGICOS

Un programa que aborde los conceptos de Diseño y Sostenibilidad como base de formación, debería tener como propósito general formar Diseñadores [de Producto] sostenibles, éticos, propositivos, con prospectiva y creativos, con la capacidad técnica, imaginativa y reflexiva para reinterpretar los procesos de diseño, producción y comercialización para transformarlos y llevarlos al campo de la. sostenibilidad, a través de los lenguajes propios de diseño.

Como apuestas pedagógicas diferenciadoras en el medio profesional se tienen:

-La creatividad como la capacidad para generar, proponer, recomponer, procesar, reestructurar y expresar las necesidades propias de nuestra época, buscando soluciones que tengan en cuenta las dimensiones de la sostenibilidad.

-La ética como línea base para su proceder profesional y cívico, que le ayude a discernir en la toma de decisiones y le ayude a construir una sociedad con equidad y sostenible.

-La ciencia, el arte y la cultura como expresión de la trascendencia y la posibilidad de identificación de una sociedad.

-La utilización de todos los medios que la creatividad, la ciencia y la tecnología ponen hoy a disposición de la formación de las disciplinas.

-El requerimiento de educadores que entiendan la docencia como un medio catalizador del conocimiento y las destrezas, en el que se reconocen y se desarrollan los diferentes imaginarios de los educandos

El plan general de estudios que se presenta, es el resultado del análisis de las tendencias y necesidades de medio. Hace parte central de la propuesta, sin embargo, se abordará desde las generalidades y se enfocará en algunos aspectos particulares que sean de significativa importancia.

Como competencias especificas del área, el programa se fundamentaría en la formación de competencias creativas, estéticas, investigativas, administrativas del campo del diseño, manejo de los lenguajes y metodologías del diseño de productos, desarrollo de pensamiento estético y manejo de herramientas tecnológicas de producción. Así mismo, centraría su actuar en la enseñanza de la condición ética y sostenible del profesional, en la que debería primar el bienestar que sus productos causen en quien los use y en el impacto que estos generen en el entorno.

Se debería entonces, concebir al diseñador de productos sostenibles bajo las siguientes competencias específicas básicas:

-Capacidad para aplicar los conceptos de diseño a productos

reales.

- Capacidad para transformar las materias primas en productos teniendo en cuenta los procesos y minimizando los impactos al medio ambiente y la sociedad.
-Habilidad para desarrollar proyectos de manera multidisciplinar, dialogando con diferentes profesiones en busca de las mejores y más éticas soluciones. • Capacidad para realizar propuestas de diseño de productos de manera ética, social y ambientalmente correctas, siendo eficientes y eficaces en el uso de los recursos que estén a disposición.

-Habilidad para solucionar problemas desde el diseño, desde el análisis crítico y conceptual de situaciones puntuales.

En definitiva, se encuentra que, para desarrollar un programa con las características planteadas, se debe formar en lo técnico, lo teórico y lo ético. Teniendo en cuenta campos del conocimiento que no son propios del diseño pero que complementarán la formación, desarrollando profesionales con amplias capacidades críticas y pragmáticas. El diseñador sostenible, tendrá que tener conocimientos en teorías y metodologías de diseño aplicadas a la sostenibilidad, estrategias de producción limpia y análisis del ciclo de vida, amplio conocimiento en nuevos materiales y procesos y un énfasis en comportamiento del consumidor. Estos serán los elementos que lo diferenciarán de los demás diseñadores y le permitirá desarrollar proyectos realmente sostenibles.

\section{CONCLUSIONES}

Con este trabajo se busca generar un impacto en el sistema educativo, haciendo un llamado para tener en cuenta el desarrollo sostenible dentro de sus políticas, no solo como discurso ambientalista, sino como postura ética, integral y contundente dentro de sus planteamientos. E componente de sostenibilidad debe ser transversal y obligatorio en cualquier programa académico y debe ser un enfoque que deben tener todas las clases de pregrado de diseño, y porque no, todos los programas académicos. Este trabajo es una invitación a los tomadores de decisiones para que asuman una postura firme y generen estrategias que permitan desarrollar en la sociedad, pensamiento crítico sobre la sostenibilidad en aspectos poco abordados como el consumo responsable y generen estilos de vida alineados con los ODS y reduzcan el impacto ambiental y social.

Se trata de una propuesta basada en el Diseño Sostenible, pero que se puede extrapolar a todo el sistema educativo, encaminando esfuerzos para que las personas asuman responsabilidades ante las consecuencias de sus acciones, así como un ejercicio a niel gubernamental, para que se amplíen las políticas públicas al respecto y tanto estados, como industria asuman compromisos reales y eficientes para alcanzar la sostenibilidad a grandes escalas.

\section{REFERENCIAS}

Alcaldía de Medellín; SIGAM. (2019). Pacto por la educación para el desarrollo sostenible con instituciones de formación superior. Medellín: Alcaldía de Medellín. 
Bakker, C., den Hollander, M., Van Hinte, E., \& Zljlstra, Y. (2014). Products that last: Product design for circular business models. TU Delft Library.

Boardman, B., Darby, S., Killip, G., Hinnells, M., Jardine, C. N., Palmer, J., \& Sinden, G. (2005). 40\% house. Oxford: Environmental Change Institute.

Bocken, N. M., \& Geradts, T. H. (2019). Barriers and drivers to sustainable business model innovation: Organization design and dynamic capabilities. Long Range Planning, 101950.

Chambouleyron, M., Arena, A. P., \& Pattini, A. (2013). Diseño de Productos Y Desarrollo Sustentable. CRICYT-CONICET, 1-6.

DANE. (2017). Cuenta ambiental y económica de flujo de materiales-residuos sólidos. Bogotá: DANE

Delgado-Huertos, E. (1998). Consumo y medio ambiente. Tabanque: Revista pedagógica, 41-66.

EAFIT. (10 de Septiembre de 2017). EAFIT: Ingenieria de diseno de producto. Obtenido de EAFIT: Ingenieria de diseno de producto: http://www.eafit.edu.co/pregrado-ingenieriadiseno-producto

Hernández-Sampieri, R. (2014). Metodología de la Investigación. México D.F.: McGRAW-HILL.

Idassarre, B., Schepers, M., Bocken, N., Cuppen, E., Korevaar, G., \& Calabretta, G. (2019). Industrial Symbiosis: towards a design process for eco-industrial clusters by integrating Circular Economy and Industrial Ecology perspectives. Journal of Cleaner Production, 446-460.

ITM. (10 de Septiembre de 2017). ITM: Ingeniería En Diseño Industrial. Obtenido de ITM: Ingeniería En Diseño Industrial: http://www.itm.edu.co/facultades/facultad-de-artes-yhumanidades-18/formacion-2/ingenieria-en-disenoindustrial-2

Mallory, R., \& Ohlman, Z. (2005). Experimental Eco-Design. Barcelona: Gustavo Gili.

Manzini, E. (2006). Design, ethics and sustainability Guidelines for a transition phase. University of Art and Design Helsinki, 9-15.

Manzini, E., \& Vezzoli, C. (2003). A strategic design approach to develop sustainable product service systems: examples taken from the 'environmentally friendly innovation' Italian prize. Journal of Cleaner Production, 851-857.

McDonough, W., \& Braungart, M. (2002). Cradle to Cradle: Remaking the Way We Make Things. New York: North Pont Press.

McDonough, W., \& Braungart, M. (2013). The Upcycle: Beyond Sustainability - Designing for Abundance. New York: North Point Press.

EN. (2003). Resolución número 3463 de 2003. Bogotá.
MEN. (2003). RESOLUCIÓN NÚMERO 3463 DE 2003. Bogotá.

MEN. (2010). Características Diseño. Bogotá: MEN.

MEN. (15 de Octubre de 2019). Creación de programas académicos. Obtenido de Creación de programas académicos: https://www.mineducacion.gov.co/1759/w3article-235796.html?_noredirect=1

MEN. (15 de Octubre de 2019). Decreto 1330 de julio 25 de 2019. Obtenido de Decreto 1330 de julio 25 de 2019: https://www.mineducacion.gov.co/1759/w3-article387348.html?_noredirect=1

MEN. (30 de 10 de 2019). Glosario de términos. Obtenido de Ministerio de Educación Nacional: https://www.mineducacion.gov.co/1621/article-79419.html

MEN. (20 de mayo de 2019). Resolución 3463 de 2003. Obtenido de Resolución 3463 de 2003: https://www.mineducacion.gov.co/1621/articles86403_Archivo_pdf.pdf

Naciones Unidas. (05 de septiembre de 2017). Objetivo 12: Garantizar modalidades de consumo y producción sostenibles. Obtenido de Objetivo 12: Garantizar modalidades de consumo y producción sostenibles: http://www.un.org/sustainabledevelopment/es/sustainableconsumption-production/

Olaya, A. (2018). Make Them Behave Sustainably. Columbia: Createspace.Williams, J. H. (2008). Employee engagement: Improving anticipation in safety. Professional Safety, 53(12), 40-45.

UdeM. (06 de 06 de 2018). Universidad de Medellín. Obtenido de Universidad de Medellín: https://www.udem.edu.co/index.php/programas-de-pregradodiseno/diseno-y-gestion-del-producto

UN - Comisión Mundial para el Medio Ambiente y el Desarrollo. (1987). Informe de la comisión mundial sobre el medio ambiente y el desarrollo: Nuestro Futuro Común. Oslo: UN

UN. (1987). Informe de la comisión mundial sobre el medio ambiente y el desarrollo: Nuestro Futuro Común. Oslo: UN

UPB. (10 de Septiembre de 2017). UPB: Diseño Industrial. Obtenido de UPB: Diseño Industrial: https://www.upb.edu.co/es/pregrados/diseno-industrialmedellin

USB Medellín. (10 de Septiembre de 2017). USB: Diseño Industrial. Obtenido de USB: Diseño Industrial: http://www.usbmed.edu.co/Programas/Pregrados/DisenoIndustrial 